Pacific Journal of Mathematics

BOTT MAPS AND THE COMPLEX PROJECTIVE PLANE:
CONSTRUCTION OF R. WOOD'S EQUIVALENCES 


\title{
BOTT MAPS AND THE COMPLEX PROJECTIVE PLANE: A CONSTRUCTION OF R. WOOD'S EQUIVALENCES
}

\author{
Minato YaSUO \\ To the memory of Dr. Shichirô Oka
}

\begin{abstract}
Let $\mathrm{U}(\infty), \mathrm{O}(\infty)$ and $\mathrm{Sp}(\infty)$ be the direct limits of the finite-dimensional unitary, orthogonal and symplectic groups under inclusion, and let $P_{2} C$ be the complex projective plane. Then, by a result of $R$. Wood in $K$-theory, there exist homotopy equivalences from $\mathrm{U}(\infty)$ to the space of based maps $\mathbf{P}_{2} \mathbf{C} \rightarrow \mathrm{O}(\infty)$, and to the space of based maps $\mathbf{P}_{2} \mathbf{C} \rightarrow \mathrm{Sp}(\infty)$. In this paper we give an explicit construction of such homotopy equivalences, and prove Wood's theorem by using classical results of $R$. Bott and elementary homotopy theory.
\end{abstract}

Introduction. It is well-known that, in topological $K$-theory, there are natural isomorphisms

$$
\widetilde{K U^{*}}(X) \rightarrow \widetilde{K \mathbf{O}^{*}}\left(X \wedge \mathbf{P}_{2} \mathbf{C}\right) \text { and } \widetilde{K U^{*}}(X) \rightarrow \widetilde{K \operatorname{Sp}^{*}}\left(X \wedge \mathbf{P}_{2} \mathbf{C}\right) \text {, }
$$

where $\mathbf{P}_{2} \mathbf{C}$ is the complex projective plane. This result is originally due to R. M. W. Wood, and his method for giving such isomorphisms can be found in [9] (see also [1; §2] or [6; §1]).

Now let $\mathrm{U}(\infty), \mathrm{O}(\infty)$ and $\mathrm{Sp}(\infty)$ be the infinite-dimensional unitary, orthogonal and symplectic groups respectively, and let $\tilde{\mathscr{C}}(X ; Y)$ denote the space of basepoint-preserving continuous maps from $X$ to $Y$ (equipped with the compact-open topology). Then the result of Wood mentioned above implies:

THEOREM (0.1) ( $R$. Wood). There are homotopy equivalences from $\mathrm{U}(\infty)$ to the space $\tilde{\mathscr{C}}\left(\mathbf{P}_{2} \mathbf{C} ; \mathrm{O}(\infty)\right)$, and to the space $\tilde{\mathscr{C}}\left(\mathbf{P}_{2} \mathbf{C} ; \mathrm{Sp}(\infty)\right)$.

The main purpose of this paper is to construct such homotopy equivalences explicitly. In $\$ 4$ we shall define certain maps

$$
\chi_{n}^{\mathrm{O}}: \mathrm{U}(2 n) \rightarrow \tilde{\mathscr{C}}\left(\mathbf{P}_{2} \mathbf{C} ; \mathrm{O}(8 n)\right) \text { and } \chi_{n}^{\mathrm{Sp}}: \mathrm{U}(n) \rightarrow \tilde{\mathscr{C}}\left(\mathbf{P}_{2} \mathbf{C} ; \mathrm{Sp}(2 n)\right) \text {, }
$$

and in $\$ 5$ we shall show (Theorem (5.4)) that these give rise to homotopy equivalences

$$
\chi_{\infty}^{\mathrm{O}}: \mathrm{U}(\infty) \rightarrow \tilde{\mathscr{C}}\left(\mathbf{P}_{2} \mathbf{C} ; \mathrm{O}(\infty)\right) \quad \text { and } \quad \chi_{\infty}^{\mathrm{Sp}}: \mathrm{U}(\infty) \rightarrow \tilde{\mathscr{C}}\left(\mathbf{P}_{2} \mathbf{C} ; \operatorname{Sp}(\infty)\right)
$$


in direct limits. Thus we shall give another proof of $(0.1)$ which does not use vector bundle theory. This work may be regarded as a continuation of [10] and [11], and indeed our proof of (0.1) is accomplished by the techniques used there. A by-product of our work is the result that, even for $n<\infty$, the maps $\chi_{n}^{\mathrm{O}}$ and $\chi_{n}^{\mathrm{Sp}}$ induce isomorphisms of homotopy groups in sufficiently low dimensions.

Throughout this paper we shall keep the notation of [10] and [11]. In particular, we denote by $\operatorname{comm}(A, B)$ the commutator $A B A^{-1} B^{-1}$.

1. Preliminaries. We begin by fixing our notation. Let $I_{n}$ be the $n \times n$ identity matrix. We put

$$
\begin{aligned}
& J_{n}=\left(\begin{array}{cc}
0 & -I_{n} \\
I_{n} & 0
\end{array}\right) \in \mathrm{SO}(2 n), \\
& T_{n}=\operatorname{diag}\left(I_{n},-I_{n}\right)=\left(\begin{array}{cc}
I_{n} & 0 \\
0 & -I_{n}
\end{array}\right) \in \mathrm{O}(2 n), \\
& K_{n}=\operatorname{diag}\left(J_{n},-J_{n}\right)=\left(\begin{array}{cccc}
0 & -I_{n} & 0 & 0 \\
I_{n} & 0 & 0 & 0 \\
0 & 0 & 0 & I_{n} \\
0 & 0 & -I_{n} & 0
\end{array}\right) \in \mathrm{SO}(4 n), \\
& S_{n}=\operatorname{diag}\left(I_{n}, J_{n} T_{n}, I_{n}\right)=\left(\begin{array}{cccc}
I_{n} & 0 & 0 & 0 \\
0 & 0 & I_{n} & 0 \\
0 & I_{n} & 0 & 0 \\
0 & 0 & 0 & I_{n}
\end{array}\right) \in \mathrm{O}(4 n) .
\end{aligned}
$$

Here $\operatorname{diag}\left(A_{1}, A_{2}, \ldots, A_{r}\right)$ denotes the square matrix with blocks $A_{1}$, $A_{2}, \ldots, A_{r}$ down the main diagonal and zeroes elsewhere. Also we let $P_{n} \in \mathrm{O}(2 n)$ be the $2 n \times 2 n$ permutation matrix defined in [10; $\left.\$ 1\right]$. This matrix represents the transformation

$$
\left(x_{1}, \ldots, x_{n}, y_{1}, \ldots, y_{n}\right) \mapsto\left(x_{1}, y_{1}, \ldots, x_{n}, y_{n}\right): \mathbf{R}^{2 n} \rightarrow \mathbf{R}^{2 n}
$$

(so that $\operatorname{det}\left(P_{n}\right)=(-1)^{n(n-1) / 2}$ ), and we put

$$
Q_{n}=P_{2 n} \operatorname{diag}\left(P_{n}, P_{n}\right) \in \mathrm{O}(4 n), \quad R_{n}=P_{4 n} \operatorname{diag}\left(Q_{n}, Q_{n}\right) \in \mathrm{SO}(8 n) .
$$

Further, as in $[10 ; \S 1]$, we put

$$
\operatorname{dec}(X+i Y)=\left(\begin{array}{cc}
X & -Y \\
Y & X
\end{array}\right), \quad \operatorname{deq}(Z+j W)=\left(\begin{array}{cc}
Z & -\bar{W} \\
W & \bar{Z}
\end{array}\right)
$$

where $X, Y$ are arbitrary $n \times n$ real matrices and $Z, W$ are arbitrary $n \times n$ complex matrices, and where $i(\in \mathbf{C})$ and $j$ are the standard generators of the algebra $\mathbf{H}$ of quaternions. 
For brevity, we write $\mathrm{O}(2 n) / \mathrm{U}=\mathrm{O}(2 n) / \mathrm{U}(n), \mathrm{U}(2 n) / \mathrm{Sp}=$ $\mathrm{U}(2 n) / \mathrm{Sp}(n), \mathrm{U}(n) / \mathrm{O}=\mathrm{U}(n) / \mathrm{O}(n)$, and $\mathrm{Sp}(n) / \mathrm{U}=\mathrm{Sp}(n) / \mathrm{U}(n)$. Here the spaces $\mathrm{O}(2 n) / \mathrm{U}(n), \mathrm{U}(2 n) / \mathrm{Sp}(n)$ are defined by using the embeddings

$$
\begin{aligned}
& A \mapsto P_{n} \operatorname{dec}(A) P_{n}^{-1}: \mathrm{U}(n) \rightarrow \mathrm{O}(2 n), \\
& A \mapsto P_{n} \operatorname{deq}(A) P_{n}^{-1}: \operatorname{Sp}(n) \rightarrow \mathrm{U}(2 n)
\end{aligned}
$$

induced by the canonical isomorphisms

$$
\begin{aligned}
& \left(x_{1}+i y_{1}, \ldots, x_{n}+i y_{n}\right) \mapsto\left(x_{1}, y_{1}, \ldots, x_{n}, y_{n}\right): \mathbf{C}^{n} \rightarrow \mathbf{R}^{2 n}, \\
& \left(z_{1}+j w_{1}, \ldots, z_{n}+j w_{n}\right) \mapsto\left(z_{1}, w_{1}, \ldots, z_{n}, w_{n}\right): \mathbf{H}^{n} \rightarrow \mathbf{C}^{2 n} .
\end{aligned}
$$

We denote by $\kappa_{n}^{\mathrm{U}}$ the latter embedding $\mathrm{Sp}(n) \rightarrow \mathrm{U}(2 n)$, by $\iota_{n}^{\mathrm{U}}$ the inclusion map $\mathrm{O}(n) \rightarrow \mathrm{U}(n)$, and by $\xi_{n}^{\mathrm{U} / \mathrm{Sp}}$ (resp. by $\xi_{n}^{\mathrm{U} / \mathrm{O}}$ ) the obvious projection map from $\mathrm{U}(2 n)$ onto $\mathrm{U}(2 n) / \mathrm{Sp}$ (resp. from $\mathrm{U}(n)$ onto $\mathrm{U}(n) / \mathrm{O})$.

Let $G$ denote either $\mathrm{O}$ or $\mathrm{Sp}$. We further put

$$
G(2 n) /(G \times G)=G(2 n) / P_{n} \operatorname{diag}(G(n) \times G(n)) P_{n}^{-1}
$$

with $\operatorname{diag}(G(n) \times G(n))=\{\operatorname{diag}(A, B) \mid A \in G(n), B \in G(n)\} \subset G(2 n)$, and write $\xi_{n}^{G /(G \times G)}$ for the projection map from $G(2 n)$ onto $G(2 n) /(G \times G)(\mathrm{cf} .[11 ; \S 1])$.

2. Bott maps for the orthogonal and symplectic groups. Here we recall classical results of Bott, which will be used in $\S 5$. Let $\Omega(X)$ denote the space of loops on $X$, and let $\Omega_{0}(X)$ denote the arcwise-connected component of the trivial loop. Consider the following maps:

$$
\begin{aligned}
& \omega_{n}^{\mathrm{O}}: \mathrm{O}(2 n) / \mathrm{U} \rightarrow \Omega(\mathrm{O}(2 n)), \quad \omega_{n}^{\mathrm{O} / \mathrm{U}}: \mathrm{U}(2 n) / \mathrm{Sp} \rightarrow \Omega(\mathrm{O}(4 n) / \mathrm{U}), \\
& \omega_{n}^{\mathrm{U} / \mathrm{Sp}}: \mathrm{Sp}(2 n) /(\mathrm{Sp} \times \mathrm{Sp}) \rightarrow \Omega_{0}(\mathrm{U}(4 n) / \mathrm{Sp}), \\
& \omega_{n}^{\mathrm{Sp} /(\mathrm{Sp} \times \mathrm{Sp})}: \mathrm{Sp}(n) \rightarrow \Omega(\mathrm{Sp}(2 n) /(\mathrm{Sp} \times \mathrm{Sp})), \\
& \omega_{n}^{\mathrm{Sp}}: \mathrm{Sp}(n) / \mathrm{U} \rightarrow \Omega(\mathrm{Sp}(n)), \quad \omega_{n}^{\mathrm{Sp} / \mathrm{U}}: \mathrm{U}(n) / \mathrm{O} \rightarrow \Omega(\mathrm{Sp}(n) / \mathrm{U}), \\
& \omega_{n}^{\mathrm{U} / \mathrm{O}}: \mathrm{O}(2 n) /(\mathrm{O} \times \mathrm{O}) \rightarrow \Omega_{0}(\mathrm{U}(2 n) / \mathrm{O}), \\
& \omega_{n}^{\mathrm{O} /(\mathrm{O} \times \mathrm{O})}: \mathrm{O}(n) \rightarrow \Omega(\mathrm{O}(2 n) /(\mathrm{O} \times \mathrm{O}))
\end{aligned}
$$

where $\omega_{n}^{\mathrm{O}}, \omega_{n}^{\mathrm{O} / \mathrm{U}}, \omega_{n}^{\mathrm{Sp}}$ and $\omega_{n}^{\mathrm{Sp} / \mathrm{U}}$ are the maps defined in [10; $\left.\$ 2\right]$, and where the maps $\omega_{n}^{\mathrm{U} / \mathrm{Sp}}, \omega_{n}^{\mathrm{U} / \mathrm{O}}, \omega_{n}^{\mathrm{O} /(\mathrm{O} \times \mathrm{O})}$ and $\omega_{n}^{\mathrm{Sp} /(\mathrm{Sp} \times \mathrm{Sp})}$ are defined as follows:

$$
\begin{aligned}
& \omega_{n}^{\mathrm{U} / \mathrm{Sp}}\left(\xi_{n}^{\mathrm{Sp} /(\mathrm{Sp} \times \mathrm{Sp})}\left(P_{n} A P_{n}^{-1}\right)\right)(t) \\
& \quad=\xi_{2 n}^{\mathrm{U} / \mathrm{Sp}}\left(Q_{n} S_{n} \exp \left(\frac{\pi}{2} t i T_{2 n}\right) S_{n} \operatorname{deq}(A) S_{n} \exp \left(-\frac{\pi}{2} t i T_{2 n}\right) S_{n} Q_{n}^{-1}\right)
\end{aligned}
$$


where $A \in \operatorname{Sp}(2 n), t \in[0,1]$

$$
\omega_{n}^{\mathrm{U} / \mathrm{O}}\left(\xi_{n}^{\mathrm{O} /(\mathrm{O} \times \mathrm{O})}\left(P_{n} A P_{n}^{-1}\right)\right)(t)=\xi_{2 n}^{\mathrm{U} / \mathrm{O}}\left(P_{n} \exp \left(\frac{\pi}{2} t i T_{n}\right) A \exp \left(-\frac{\pi}{2} t i T_{n}\right) P_{n}^{-1}\right)
$$

where $A \in \mathrm{O}(2 n), t \in[0,1]$;

$$
\omega_{n}^{G /(G \times G)}(A)(t)=\xi_{n}^{G /(G \times G)}\left(P_{n} \exp \left(\frac{\pi}{2} t J_{n}\right) \operatorname{diag}\left(A, I_{n}\right) \exp \left(-\frac{\pi}{2} t J_{n}\right) P_{n}^{-1}\right)
$$

where $A \in G(n), t \in[0,1]$, and $G=\mathrm{O}$ or $\mathrm{Sp}$ as in $\S 1$. Then the direct limit maps

$$
\omega_{\infty}^{\mathrm{O}}: \mathrm{O}(\infty) / \mathrm{U} \rightarrow \Omega(\mathrm{O}(\infty)), \omega_{\infty}^{\mathrm{O} / \mathrm{U}}: \mathrm{U}(\infty) / \mathrm{Sp} \rightarrow \Omega(\mathrm{O}(\infty) / \mathrm{U}), \text { etc. }
$$

where we have put $\omega_{\infty}^{\mathrm{O}}=\lim _{\rightarrow \rightarrow} \omega_{n}^{\mathrm{O}}, \mathrm{O}(\infty) / \mathrm{U}=\lim _{\rightarrow} \mathrm{O}(2 n) / \mathrm{U}$, etc., are defined in the usual way, ${ }^{1} \overrightarrow{\text { and }}$ the Bott periodicity theorems for the orthogonal and symplectic groups are immediate consequences of the following:

TheOREM (2.1) (see [2], [3], [4], [5], and also [8; \$24]). The maps $\omega_{\infty}^{\mathrm{O}}$, $\omega_{\infty}^{\mathrm{O} / \mathrm{U}}, \omega_{\infty}^{\mathrm{U} / \mathrm{Sp}}, \omega_{\infty}^{\mathrm{Sp} /(\mathrm{Sp} \times \mathrm{Sp})}, \omega_{\infty}^{\mathrm{Sp}}, \omega_{\infty}^{\mathrm{Sp} / \mathrm{U}}, \omega_{\infty}^{\mathrm{U} / \mathrm{O}}$ and $\omega_{\infty}^{\mathrm{O} /(\mathrm{O} \times \mathrm{O})}$ are homotopy equivalences.

3. The maps $\nu_{n}^{\mathrm{U} / \mathrm{Sp}}$ and $\nu_{n}^{\mathrm{U} / \mathrm{O}}$. For later use, we define here the maps $\nu_{n}^{\mathrm{U} / \mathrm{Sp}}: \mathrm{U}(2 n) / \mathrm{Sp} \rightarrow \mathrm{U}(4 n) / \mathrm{Sp}$ and $\nu_{n}^{\mathrm{U} / \mathrm{O}}: \mathrm{U}(n) / \mathrm{O} \rightarrow \mathrm{U}(2 n) / \mathrm{O}$ as follows:

$$
\begin{gathered}
\nu_{n}^{\mathrm{U} / \mathrm{Sp}}\left(\xi_{n}^{\mathrm{U} / \mathrm{Sp}}\left(P_{n} A P_{n}^{-1}\right)\right)=\xi_{2 n}^{\mathrm{U} / \mathrm{Sp}}\left(Q_{n} S_{n} \operatorname{diag}\left(A, I_{2 n}\right) S_{n} Q_{n}^{-1}\right) \quad \text { for } A \in \mathrm{U}(2 n) ; \\
\nu_{n}^{\mathrm{U} / \mathrm{O}}\left(\xi_{n}^{\mathrm{U} / \mathrm{O}}(A)\right)=\xi_{2 n}^{\mathrm{U} / \mathrm{O}}\left(P_{n} \operatorname{diag}\left(A, I_{n}\right) P_{n}^{-1}\right) \quad \text { for } A \in \mathrm{U}(n) .
\end{gathered}
$$

Consider now the direct limits $\nu_{\infty}^{\mathrm{U} / \mathrm{Sp}}=\lim _{\rightarrow} \nu_{n}^{\mathrm{U} / \mathrm{Sp}}$ and $\nu_{\infty}^{\mathrm{U} / \mathrm{O}}=\lim _{\rightarrow} \nu_{n}^{\mathrm{U} / \mathrm{O}}$. Then by an elementary argument used in $[\mathbf{5 ;} \S 1]$, we can see:

LEMMA (3.1). The map $\nu_{\infty}^{\mathrm{U} / \mathrm{Sp}}\left(\right.$ resp. $\left.\nu_{\infty}^{\mathrm{U} / \mathrm{O}}\right)$ is homotopic to the identity map of $\mathrm{U}(\infty) / \mathrm{Sp}$ (resp. of $\mathrm{U}(\infty) / \mathrm{O})$.

For a proof, see Appendix 1. An immediate consequence of this lemma is that $\nu_{\infty}^{\mathrm{U} / \mathrm{Sp}}$ and $\nu_{\infty}^{\mathrm{U} / \mathrm{O}}$ are homotopy (self-) equivalences. We shall use this fact in $\S 5$.

${ }^{1}$ Strictly speaking, for example $\omega_{\infty}^{\mathrm{O}}$ is defined as the composition of the dierct limit map $\lim _{\rightarrow} \omega_{n}^{\mathrm{O}}: \underset{\rightarrow}{\lim } \mathrm{O}(2 n) / \mathrm{U} \rightarrow \underset{\rightarrow}{\lim } \Omega(\mathrm{O}(2 n))$ and the canonical bijection $\stackrel{\lim }{\rightarrow} \Omega(\mathrm{O}(2 n)) \rightarrow$ $\overrightarrow{\Omega(}\left(\lim _{\rightarrow} \mathrm{O}(\overrightarrow{2 n})\right)$. But here and throughout we simply write $\omega_{\infty}^{\mathrm{O}}=\lim _{\rightarrow} \omega_{n}^{\mathrm{O}}$, etc., by abuse of notation. 
4. Definition of the maps $\chi_{n}^{\mathrm{O}}$ and $\chi_{n}^{\mathrm{Sp}}$. We continue to use the notation of $\S 1$. For each $\left(z_{0}, z_{1}, z_{2}\right) \in \mathbf{C}^{3}$, let us now put

$$
L_{n}\left(z_{1}, z_{2}\right)=\operatorname{diag}\left(z_{1} I_{n}, \bar{z}_{1} I_{n}\right)+z_{2} i T_{n} J_{n}=\left(\begin{array}{cc}
z_{1} I_{n} & -z_{2} i I_{n} \\
-z_{2} i I_{n} & \bar{z}_{1} I_{n}
\end{array}\right),
$$

$M_{n}\left(z_{0}, z_{1}, z_{2}\right)=\operatorname{dec}\left(S_{n} z_{0} I_{4 n} S_{n}\right)+K_{2 n} \operatorname{dec}\left(S_{n} L_{2 n}\left(z_{1}, z_{2}\right) S_{n}\right)$

$=\left(\begin{array}{cc|cc|cc|cc}x_{0} I_{n} & 0 & -x_{1} I_{n} & -y_{2} I_{n} & -y_{0} I_{n} & 0 & y_{1} I_{n} & -x_{2} I_{n} \\ 0 & x_{0} I_{n} & -y_{2} I_{n} & -x_{1} I_{n} & 0 & -y_{0} I_{n} & -x_{2} I_{n} & -y_{1} I_{n} \\ \hline x_{1} I_{n} & y_{2} I_{n} & x_{0} I_{n} & 0 & -y_{1} I_{n} & x_{2} I_{n} & -y_{0} I_{n} & 0 \\ y_{2} I_{n} & x_{1} I_{n} & 0 & x_{0} I_{n} & x_{2} I_{n} & y_{1} I_{n} & 0 & -y_{0} I_{n} \\ \hline y_{0} I_{n} & 0 & y_{1} I_{n} & -x_{2} I_{n} & x_{0} I_{n} & 0 & x_{1} I_{n} & y_{2} I_{n} \\ 0 & y_{0} I_{n} & -x_{2} I_{n} & -y_{1} I_{n} & 0 & x_{0} I_{n} & y_{2} I_{n} & x_{1} I_{n} \\ \hline-y_{1} I_{n} & x_{2} I_{n} & y_{0} I_{n} & 0 & -x_{1} I_{n} & -y_{2} I_{n} & x_{0} I_{n} & 0 \\ x_{2} I_{n} & y_{1} I_{n} & 0 & y_{0} I_{n} & -y_{2} I_{n} & -x_{1} I_{n} & 0 & x_{0} I_{n}\end{array}\right)$,

$$
N_{n}\left(z_{0}, z_{1}, z_{2}\right)=z_{0} I_{2 n}+j L_{n}\left(z_{1}, z_{2}\right)=\left(\begin{array}{cc}
\left(z_{0}+j z_{1}\right) I_{n} & i j z_{2} I_{n} \\
i j z_{2} I_{n} & \left(z_{0}+j \bar{z}_{1}\right) I_{n}
\end{array}\right)
$$

with $z_{r}=x_{r}+i y_{r}, x_{r} \in \mathbf{R}, y_{r} \in \mathbf{R}(r=0,1,2)$, and consider the unit 4-sphere

$$
\mathbf{S}\left(\mathbf{C}^{2} \times \mathbf{R}\right)=\left\{\left(w_{0}, w_{1}, w_{2}\right) \in \mathbf{S}\left(\mathbf{C}^{3}\right) \mid w_{2} \in \mathbf{R}\right\}
$$

where

$$
\mathbf{S}\left(\mathbf{C}^{3}\right)=\left\{\left.\left(w_{0}, w_{1}, w_{2}\right) \in \mathbf{C}^{3}|| w_{0}\right|^{2}+\left|w_{1}\right|^{2}+\left|w_{2}\right|^{2}=1\right\} .
$$

Then we can see by elementary calculations that

$$
M_{n}\left(w_{0}, w_{1}, w_{2}\right) \in \mathrm{O}(8 n) \text { and } N_{n}\left(w_{0}, w_{1}, w_{2}\right) \in \mathrm{Sp}(2 n)
$$

for all $\left(w_{0}, w_{1}, w_{2}\right) \in \mathbf{S}\left(\mathbf{C}^{2} \times \mathbf{R}\right)$. Bearing this in mind, we define the maps $\chi_{n}^{\mathrm{O}}$ and $\chi_{n}^{\mathrm{Sp}}$ mentioned in the introduction, as follows:

If $\left(w_{0}, w_{1}, w_{2}\right) \in \mathbf{S}\left(\mathbf{C}^{2} \times \mathbf{R}\right)$, then we put

$$
\begin{aligned}
& \chi_{n}^{\mathrm{O}}\left(P_{n} A P_{n}^{-1}\right)\left(\left[w_{0}: w_{1}: w_{2}\right]\right) \\
& \quad=R_{n} \operatorname{comm}\left(M_{n}\left(w_{0}, w_{1}, w_{2}\right), \operatorname{dec}\left(S_{n} \operatorname{diag}\left(A, I_{2 n}\right) S_{n}\right)\right) R_{n}^{-1}
\end{aligned}
$$

for $A \in \mathrm{U}(2 n)$, and

$$
\chi_{n}^{\mathrm{Sp}}(A)\left(\left[w_{0}: w_{1}: w_{2}\right]\right)=P_{n} \operatorname{comm}\left(N_{n}\left(w_{0}, w_{1}, w_{2}\right), \operatorname{diag}\left(A, I_{n}\right)\right) P_{n}^{-1}
$$

for $A \in \mathrm{U}(n)$. If $\left(w_{0}, w_{1}, w_{2}\right) \in \mathbf{S}\left(\mathbf{C}^{3}\right)$ and $w_{2} \neq 0$, then we put

$$
\begin{aligned}
& \chi_{n}^{\mathrm{O}}\left(P_{n} A P_{n}^{-1}\right)\left(\left[w_{0}: w_{1}: w_{2}\right]\right) \\
& \quad=\chi_{n}^{\mathrm{O}}\left(P_{n} A P_{n}^{-1}\right)\left(\left[w_{0} \bar{w}_{2} /\left|w_{2}\right|: w_{1} \bar{w}_{2} /\left|w_{2}\right|:\left|w_{2}\right|\right]\right)
\end{aligned}
$$


for $A \in \mathrm{U}(2 n)$, and

$$
\chi_{n}^{\mathrm{Sp}}(A)\left(\left[w_{0}: w_{1}: w_{2}\right]\right)=\chi_{n}^{\mathrm{Sp}}(A)\left(\left[w_{0} \bar{w}_{2} /\left|w_{2}\right|: w_{1} \bar{w}_{2} /\left|w_{2}\right|:\left|w_{2}\right|\right]\right)
$$

for $A \in \mathrm{U}(n)$. Here $\left[w_{0}: w_{1}: w_{2}\right]$ denotes the point of $\mathbf{P}_{2} \mathbf{C}$ corresponding to $\left(w_{0}, w_{1}, w_{2}\right) \in \mathbf{S}\left(\mathbf{C}^{3}\right)$.

We leave it to the reader to check that $\chi_{n}^{\mathrm{O}}$ and $\chi_{n}^{\mathrm{Sp}}$ are well-defined.

5. The main theorem. As before let $\tilde{\mathscr{C}}(X ; Y)$ denote the space of based maps $X \rightarrow Y$. Henceforth we use the following conventions (see also Appendix 2):

(1) Let $\mathbf{P}_{1} \mathbf{C}=\left\{\left[z_{0}: z_{1}\right] \mid\left(z_{0}, z_{1}\right) \in \mathbf{C}^{2},\left(z_{0}, z_{1}\right) \neq(0,0)\right\}$ be the complex projective line. Then each element $f$ of $\tilde{\mathscr{C}}\left(\mathbf{P}_{1} \mathbf{C} ; Y\right)$ is regarded as an element of $\Omega^{2}(Y)=\Omega(\Omega(Y))$ by putting

$$
f(u)(v)=f([\cos (\pi v)+i \sin (\pi v) \cos (\pi u): \sin (\pi v) \sin (\pi u)])
$$

for $u, v \in[0,1]$. In this way we identify $\tilde{\mathscr{C}}\left(\mathbf{P}_{1} \mathbf{C} ; Y\right)$ with the double loop space of $Y$.

(2) Also we identify $\tilde{\mathscr{C}}\left(\mathbf{P}_{2} \mathbf{C} / \mathbf{P}_{1} \mathbf{C} ; Y\right)$ with the 4 th iterated loop space of $Y$ in the following way: Let $q: \mathbf{P}_{2} \mathbf{C} \rightarrow \mathbf{P}_{2} \mathbf{C} / \mathbf{P}_{1} \mathbf{C}$ be the canonical map, and let

$(*)\left\{\begin{array}{l}w_{0}(u, v)=\cos (\pi v)+i \sin (\pi v) \cos (\pi u), \\ w_{1}(s, t, u, v)=\sin (\pi v) \sin (\pi u)(\cos (\pi t)+i \sin (\pi t) \cos (\pi s)), \\ w_{2}(s, t, u, v)=\sin (\pi v) \sin (\pi u) \sin (\pi t) \sin (\pi s) .\end{array}\right.$

Then each $g \in \tilde{\mathscr{C}}\left(\mathbf{P}_{2} \mathbf{C} / \mathbf{P}_{1} \mathbf{C} ; Y\right)$ is regarded as an element of $\Omega^{4}(Y)$ by

$$
g(s)(t)(u)(v)=g\left(q\left(\left[w_{0}(u, v): w_{1}(s, t, u, v): w_{2}(s, t, u, v)\right]\right)\right) .
$$

With these understood, consider now the diagrams

$(5.1)_{n}$

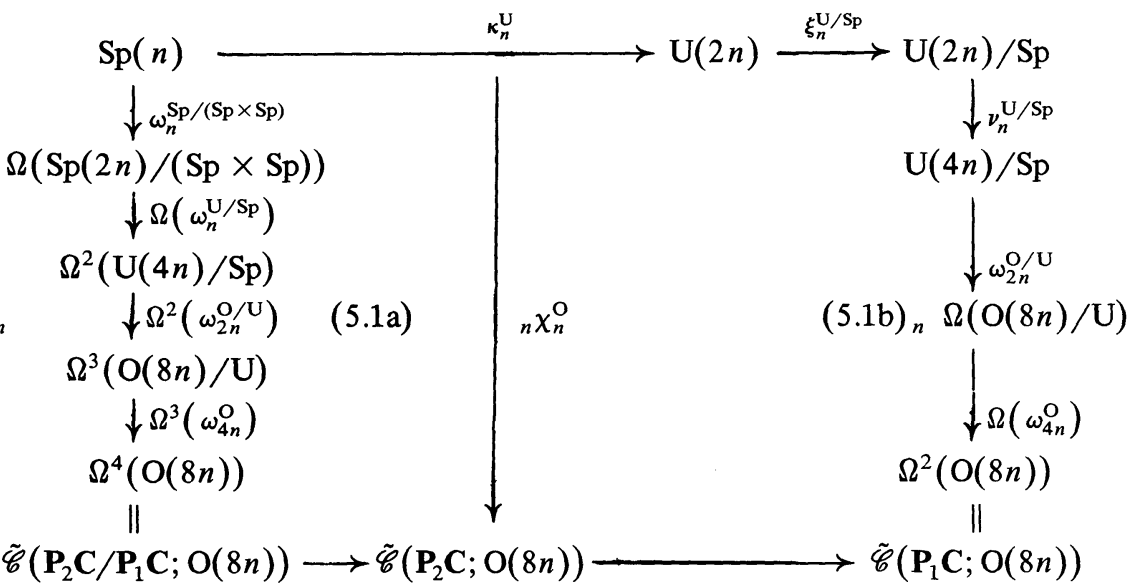


and

$(5.2)_{n}$

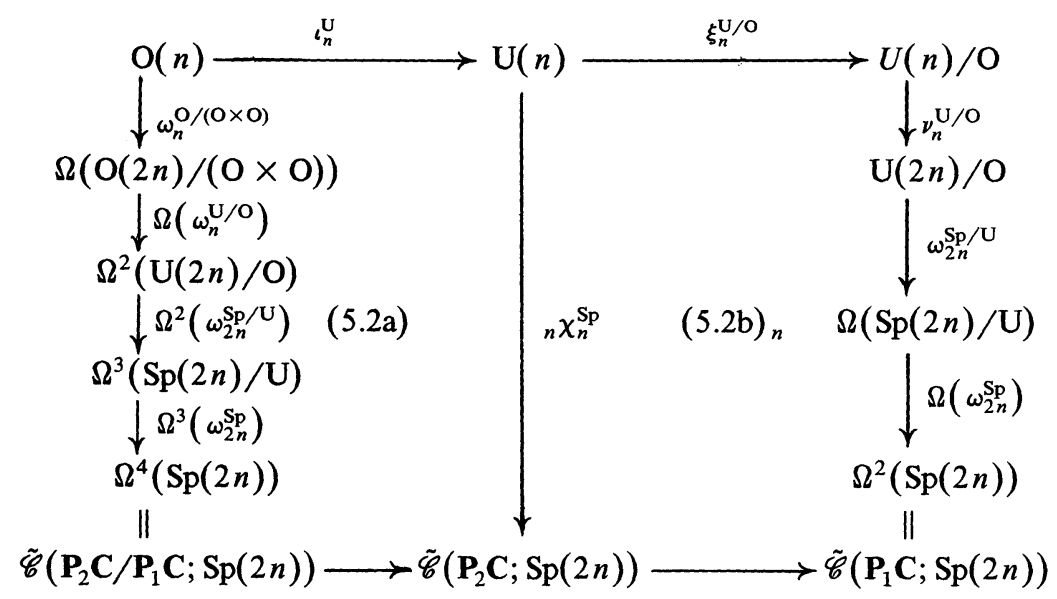

where the labelled maps are as defined before and the bottom rows are induced by the obvious cofibration $\mathbf{P}_{2} \mathbf{C} / \mathbf{P}_{1} \mathbf{C} \leftarrow \mathbf{P}_{2} \mathbf{C} \leftarrow \mathbf{P}_{1} \mathbf{C}$. Taking the direct limits and writing $\chi_{\infty}^{\mathrm{O}}=\lim _{\rightarrow} \chi_{n}^{\mathrm{O}}, \chi_{\infty}^{\mathrm{Sp}}=\lim _{\rightarrow} \chi_{n}^{\mathrm{Sp}}$, etc., we then get the diagrams $(5.1)_{n}$ and $(5.2)_{n}$ for $n=\infty$, in which all rows are (Hurewicz) fibration sequences.

Proposition (5.3). The diagrams $(5.1)_{n}$ and $(5.2)_{n}$ for $n \leq \infty$ are homotopy-commutative.

This will be proved in $\S 6$, the next section. Our main theorem is the following, which is a refinement of Theorem (0.1):

THEOREM (5.4). The maps $\chi_{\infty}^{\mathrm{O}}$ and $\chi_{\infty}^{\mathrm{Sp}}$ are homotopy equivalences, and:

(i) the homomorphism $\left(\chi_{n}^{O}\right)_{*}: \pi_{r}(\mathrm{U}(2 n)) \rightarrow \pi_{r}\left(\tilde{\mathscr{C}}\left(\mathbf{P}_{2} \mathbf{C} ; \mathrm{O}(8 n)\right)\right)$ induced by $\chi_{n}^{\mathrm{O}}$ is isomorphic for $r \leq 4 n-1$ with $(r, n) \neq(3,1)$;

(ii) the homomorphism $\left(\chi_{n}^{\mathrm{Sp}}\right)_{*}: \pi_{r}(U(n)) \rightarrow \pi_{r}\left(\tilde{\mathscr{C}}\left(\mathbf{P}_{2} \mathbf{C} ; \mathrm{Sp}(2 n)\right)\right)$ induced by $\chi_{n}^{\mathrm{Sp}}$ is isomorphic for $r \leq 2 n-1$.

Proof. The part for $n=\infty$ is obtained by an easy five-lemma argument: Combining Theorem (2.1), Lemma (3.1) and Proposition (5.3), and noting J. H. C. Whitehead's theorem (and Theorem 3 of [7]), we see that $\chi_{\infty}^{\mathrm{O}}$ and $\chi_{\infty}^{\mathrm{Sp}}$ are homotopy equivalences. 
The remaining part is proved as follows. ${ }^{2}$ Consider the commutative diagram

$$
\begin{array}{ccc}
\pi_{r}(\mathrm{U}(\infty)) & \stackrel{\left(\chi_{\infty}^{\mathrm{o}}\right)_{*}}{\rightarrow} & \pi_{r}\left(\tilde{\mathscr{C}}\left(\mathbf{P}_{2} \mathrm{C} ; \mathrm{O}(\infty)\right)\right) \\
\uparrow & & \uparrow \\
\pi_{r}(\mathrm{U}(2 n)) & \stackrel{\left(\chi_{n}^{\mathrm{o}}\right)_{*}}{\rightarrow} & \pi_{r}\left(\tilde{\mathscr{C}}\left(\mathbf{P}_{2} \mathbf{C} ; \mathrm{O}(8 n)\right)\right)
\end{array}
$$

where the verticals are the canonical homomorphisms. Then the left-hand vertical is an isomorphism for $r \leq 4 n-1$, while the right-hand vertical is an isomorphism for $r \leq 8 n-6$. (Note that $(\mathrm{O}(\infty), \mathrm{O}(8 n))$ is $(8 n-1)$ connected.) Hence (i) follows. The assertion (ii) can be verified analogously.

REMARK. One can easily check that for $(r, n)=(3,1)$ the homomorphism $\left(\chi_{1}^{\mathrm{O}}\right)_{*}: \pi_{3}(\mathrm{U}(2)) \rightarrow \pi_{3}\left(\tilde{\mathscr{C}}\left(\mathbf{P}_{2} \mathbf{C} ; \mathrm{O}(8)\right)\right)$ is monomorphic but not epimorphic.

6. Proof of Proposition (5.3). First we shall show that the subdiagrams $(5.1 b)_{n}$ and $(5.2 b)_{n}$ are homotopy-commutative. For this, consider the maps

$$
\Theta_{2 n}^{\mathrm{O}}(r): \mathrm{U}(4 n) / \mathrm{Sp} \rightarrow \Omega^{2}(\mathrm{O}(8 n)) \text { and } \Theta_{2 n}^{\mathrm{Sp}}(r): \mathrm{U}(2 n) / \mathrm{O} \rightarrow \Omega^{2}(\mathrm{Sp}(2 n))
$$

defined in [10; §4], where $r \in[0,1]$. If in $(5.1 b)_{n}$ and $(5.2 b)_{n}$ we replace the map

$$
\Omega\left(\omega_{4 n}^{\mathrm{O}}\right) \circ \omega_{2 n}^{\mathrm{O} / \mathrm{U}}: \mathrm{U}(4 n) / \mathrm{Sp} \rightarrow \Omega(\mathrm{O}(8 n) / \mathrm{U}) \rightarrow \Omega^{2}(\mathrm{O}(8 n))
$$

by $\Theta_{2 n}^{\mathrm{O}}(0)$ and the map

$$
\Omega\left(\omega_{2 n}^{\mathrm{Sp}}\right) \circ \omega_{2 n}^{\mathrm{Sp} / \mathrm{U}}: \mathrm{U}(2 n) / \mathrm{O} \rightarrow \Omega(\mathrm{Sp}(2 n) / \mathrm{U}) \rightarrow \Omega^{2}(\mathrm{Sp}(2 n))
$$

by $\Theta_{2 n}^{\mathrm{Sp}}(0)$ respectively, then the resulting diagrams are strictly commutative, as seen by direct calculations. On the other hand, as mentioned in $[10 ; \S 4]$, we have

$$
\Theta_{2 n}^{\mathrm{O}}(1)=\Omega\left(\omega_{4 n}^{\mathrm{O}}\right) \circ \omega_{2 n}^{\mathrm{O} / \mathrm{U}} \text { and } \Theta_{2 n}^{\mathrm{Sp}}(1)=\Omega\left(\omega_{2 n}^{\mathrm{Sp}}\right) \circ \omega_{2 n}^{\mathrm{Sp} / \mathrm{U}} .
$$

Hence the homotopy-commutativity of $(5.1 \mathrm{~b})_{n}$ and $(5.2 \mathrm{~b})_{n}$ for $n<\infty$ follows, and considering the direct limits $\Theta_{\infty}^{\mathrm{O}}(r)$ and $\Theta_{\infty}^{\mathrm{Sp}}(r)$, we see that $(5.1 b)_{\infty}$ and $(5.2 b)_{\infty}$ are also homotopy-commutative.

\footnotetext{
${ }^{2}$ This proof was communicated to the author by S. Oka.
} 
Next we shall prove the homotopy-commutativity of $(5.1 \mathrm{a})_{n}$ and $(5.2 \mathrm{a})_{n}$. For $r, s, t, u, v \in[0,1]$, let

$$
F_{2 n}(r, u, v) \in \mathrm{O}(8 n) \text { and } G_{2 n}(r, u, v) \in \mathrm{Sp}(2 n)
$$

be as defined in $[10 ; \S 4]$, and put

$$
\begin{aligned}
& V_{n}(s, t, u)=\exp \left(\frac{\pi}{2} u K_{2 n}\right) \operatorname{dec}\left(S_{n} \exp \left(\frac{\pi}{2} t i T_{2 n}\right) \exp \left(\frac{\pi}{2} s J_{2 n}\right) S_{n}\right) \in \mathrm{O}(8 n), \\
& W_{n}(s, t, u)=\exp \left(\frac{\pi}{2} u j I_{2 n}\right) \exp \left(\frac{\pi}{2} t i T_{n}\right) \exp \left(\frac{\pi}{2} s J_{n}\right) \in \operatorname{Sp}(2 n) .
\end{aligned}
$$

Further, put $V_{n}(s, t)=V_{n}(s, t, 0), W_{n}(s, t)=W_{n}(s, t, 0)$, and define the maps

$$
\Pi_{n}^{\mathrm{O}}(r): \operatorname{Sp}(n) \rightarrow \Omega^{4}(\mathrm{O}(8 n)) \quad \text { and } \quad \Pi_{n}^{\mathrm{Sp}}(r): \mathrm{O}(n) \rightarrow \Omega^{4}(\operatorname{Sp}(2 n))
$$

for each $r \in[0,1]$, as follows:

$$
\begin{aligned}
& \Pi_{n}^{\mathrm{O}}(r)(A)(s)(t)(u)(v) \\
& \quad=R_{n} V_{n}(r s, r t, r u) C_{n}(A ; r, s, t, u, v)\left(V_{n}(r s, r t, r u)\right)^{-1} R_{n}^{-1}
\end{aligned}
$$

where $A \in \operatorname{Sp}(n)$ and

$$
\begin{gathered}
C_{n}(A ; r, s, t, u, v) \\
=\operatorname{comm}\left(\left(V_{n}(s, t)\right)^{-1} F_{2 n}(r, u, v) V_{n}(s, t), \operatorname{dec}\left(S_{n} \operatorname{diag}\left(\operatorname{deq}(A), I_{2 n}\right) S_{n}\right)\right) \\
\Pi_{n}^{\mathrm{Sp}}(r)(A)(s)(t)(u)(v) \\
=P_{n} W_{n}(r s, r t, r u) D_{n}(A ; r, s, t, u, v)\left(W_{n}(r s, r t, r u)\right)^{-1} P_{n}^{-1}
\end{gathered}
$$

where $A \in \mathrm{O}(n)$ and

$$
\begin{aligned}
& D_{n}(A ; r, s, t, u, v) \\
& \quad=\operatorname{comm}\left(\left(W_{n}(s, t)\right)^{-1} G_{2 n}(r, u, v) W_{n}(s, t), \operatorname{diag}\left(A, I_{n}\right)\right) .
\end{aligned}
$$

Then for $r=0$, we have

$$
\begin{aligned}
& F_{2 n}(0, u, v)=I_{8 n} \cos (\pi v)+J_{4 n} \sin (\pi v) \cos (\pi u)+K_{2 n} \sin (\pi v) \sin (\pi u), \\
& G_{2 n}(0, u, v)=I_{2 n} \cos (\pi v)+i I_{2 n} \sin (\pi v) \cos (\pi u)+j I_{2 n} \sin (\pi v) \sin (\pi u),
\end{aligned}
$$

and calculations show that

$$
\begin{aligned}
& \left(V_{n}(s, t)\right)^{-1} F_{2 n}(0, u, v) V_{n}(s, t) \\
& \quad=M_{n}\left(w_{0}(u, v), w_{1}(s, t, u, v), w_{2}(s, t, u, v)\right), \\
& \left(W_{n}(s, t)\right)^{-1} G_{2 n}(0, u, v) W_{n}(s, t) \\
& \quad=N_{n}\left(w_{0}(u, v), w_{1}(s, t, u, v), w_{2}(s, t, u, v)\right)
\end{aligned}
$$


where $w_{0}(u, v), w_{1}(s, t, u, v)$ and $w_{2}(s, t, u, v)$ are given by the formulae (*) at the beginning of $\S 5$ and where $M_{n}\left(z_{0}, z_{1}, z_{2}\right)$ and $N_{n}\left(z_{0}, z_{1}, z_{2}\right)$ are as defined in $\S 4$. Hence we see that the map $\chi_{n}^{\mathrm{O}} \circ \kappa_{n}^{\mathrm{U}}$ is just the composite map

$$
\mathrm{Sp}(n) \stackrel{\Pi_{n}^{\circ}(0)}{\rightarrow} \Omega^{4}(\mathrm{O}(8 n))=\tilde{\mathscr{C}}\left(\mathbf{P}_{2} \mathbf{C} / \mathbf{P}_{1} \mathbf{C} ; \mathrm{O}(8 n)\right) \rightarrow \tilde{\mathscr{C}}\left(\mathbf{P}_{2} \mathbf{C} ; \mathrm{O}(8 n)\right)
$$

and the $\operatorname{map} \chi_{n}^{\mathrm{Sp}} \circ \iota_{n}^{\mathrm{U}}$ is equal to the composition

$$
\mathrm{O}(n) \stackrel{\Pi_{n}^{\mathrm{Sp}}(0)}{\rightarrow} \Omega^{4}(\operatorname{Sp}(2 n))=\tilde{\mathscr{C}}\left(\mathbf{P}_{2} \mathbf{C} / \mathbf{P}_{1} \mathbf{C} ; \operatorname{Sp}(2 n)\right) \rightarrow \tilde{\mathscr{C}}\left(\mathbf{P}_{2} \mathbf{C} ; \operatorname{Sp}(2 n)\right)
$$

(where the unlabelled arrows are the maps induced by the canonical surjection $\left.\mathbf{P}_{2} \mathbf{C} \rightarrow \mathbf{P}_{2} \mathbf{C} / \mathbf{P}_{1} \mathbf{C}\right)$. Also, noting the equalities

$$
\begin{aligned}
& \left(V_{n}(s, t)\right)^{-1} F_{2 n}(1, u, v) V_{n}(s, t) \\
& \quad=\left(V_{n}(s, t, u)\right)^{-1} \exp \left(\pi v J_{4 n}\right) V_{n}(s, t, u), \\
& \left(W_{n}(s, t)\right)^{-1} G_{2 n}(1, u, v) W_{n}(s, t) \\
& \quad=\left(W_{n}(s, t, u)\right)^{-1} \exp \left(\pi v i I_{2 n}\right) W_{n}(s, t, u),
\end{aligned}
$$

we see by calculations that

$$
\begin{aligned}
& \Pi_{n}^{\mathrm{O}}(1)=\Omega^{3}\left(\omega_{4 n}^{\mathrm{O}}\right) \circ \Omega^{2}\left(\omega_{2 n}^{\mathrm{O} / \mathrm{U}}\right) \circ \Omega\left(\omega_{n}^{\mathrm{U} / \mathrm{Sp}}\right) \circ \omega_{n}^{\mathrm{Sp} /(\mathrm{Sp} \times \mathrm{Sp})}, \\
& \Pi_{n}^{\mathrm{Sp}}(1)=\Omega^{3}\left(\omega_{2 n}^{\mathrm{Sp}}\right) \circ \Omega^{2}\left(\omega_{2 n}^{\mathrm{Sp} / \mathrm{U}}\right) \circ \Omega\left(\omega_{n}^{\mathrm{U} / \mathrm{O}}\right) \circ \omega_{n}^{\mathrm{O} /(\mathrm{O} \times \mathrm{O})} .
\end{aligned}
$$

Hence the homotopy-commutativity of $(5.1 \mathrm{a})_{n}$ and $(5.2 \mathrm{a})_{n}$ for $n<\infty$ is clear, and considering $\Pi_{\infty}^{\mathrm{O}}(r)$ and $\Pi_{\infty}^{\mathrm{Sp}}(r)$, we conclude that $(5.1 \mathrm{a})_{\infty}$ and $(5.2 \mathrm{a})_{\infty}$ are also homotopy-commutative.

Appendix 1. Proof of Lemma (3.1). For completeness we record a proof of (3.1) here. ${ }^{3}$ First, choose a path $\Lambda_{n}:[0,1] \rightarrow \mathrm{SO}(n+2)$ for each $n$ so that $\Lambda_{n}(0)=I_{n+2}$ and $\Lambda_{n}(1)$ is the permutation matrix associated to the 3-cycle: $1 \mapsto n+1, n+1 \mapsto n+2, n+2 \mapsto 1$. Further, define $\Gamma_{n}(t)$ $\in \mathrm{SO}(2 n)$ inductively by

$$
\Gamma_{1}(t)=I_{2} \quad \text { and } \quad \Gamma_{n+1}(t)=\operatorname{diag}\left(\Gamma_{n}(t), I_{2}\right) \operatorname{diag}\left(I_{n}, \Lambda_{n}(t)\right),
$$

where $t \in[0,1]$. Note that $\Gamma_{n}(1)$ is a $2 n \times 2 n$ permutation matrix and the corresponding permutation takes $r$ to $2 r-1$ for $1 \leq r \leq n$.

\footnotetext{
${ }^{3}$ The author learned the techniques of this proof from Chapter $4, \S 3$ of the following book: H. Toda and M. Mimura, The topology of Lie groups (Japanese), Vol. 1, Kinokuniya Sûgaku Sôsho 14-A, Kinokuniya Book-Store, Tokyo, 1978.
} 
It is now easy to see that $\nu_{\infty}^{\mathrm{U} / \mathrm{O}}$ is homotopic to the identity map: Consider the family of maps

$$
A \mapsto \Gamma_{n}(t) \operatorname{diag}\left(A, I_{n}\right)\left(\Gamma_{n}(t)\right)^{-1}: \mathrm{U}(n) \rightarrow \mathrm{U}(2 n) \quad(t \in[0,1]) .
$$

By passage to the quotients, these induce maps $\mathrm{U}(n) / \mathrm{O} \rightarrow \mathrm{U}(2 n) / \mathrm{O}$, and then, since $\Gamma_{n}(1) \operatorname{diag}\left(A, I_{n}\right)\left(\Gamma_{n}(1)\right)^{-1}=P_{n} \operatorname{diag}\left(A, I_{n}\right) P_{n}^{-1}$ and $\Gamma_{n}(0)=I_{2 n}$, we get a homotopy between $\nu_{n}^{\mathrm{U} / \mathrm{O}}$ and the canonical injection $\mathrm{U}(n) / \mathrm{O} \rightarrow$ $\mathrm{U}(2 n) / \mathrm{O}$ for each $n$. Taking the direct limit, we get the required homotopy.

Replacing $\mathrm{U}(n) / \mathrm{O}$ by $\mathrm{U}(2 n) / \mathrm{Sp}$, and $\Gamma_{n}(t)$ by the Kronecker product of $\Gamma_{n}(t)$ and $I_{2}$, we can see by the same type of argument that $\nu_{\infty}^{\mathrm{U} / \mathrm{Sp}}$ is homotopic to the identity. We leave further details to the reader.

Appendix 2. Note on the conventions mentioned in \$5. For brevity we let $I=[0,1]$ here. Let $\mathbf{P}_{n} \mathbf{C}$ be the $n$-dimensional complex projective space, and let $Y$ be an arbitrary based space. In $\S 5$, we have identified the space $\tilde{\mathscr{C}}\left(\mathbf{P}_{1} \mathbf{C} ; Y\right)$ with $\Omega^{2}(Y)$ and the space $\tilde{\mathscr{C}}\left(\mathbf{P}_{2} \mathbf{C} / \mathbf{P}_{1} \mathbf{C} ; Y\right)$ with $\Omega^{4}(Y)$. These identifications are based on the following observations:

(1) Let $\mathbf{P}_{m} \mathbf{R}$ be the $m$-dimensional real projective space, and put

$$
\begin{aligned}
& u_{0}=\cos \left(\pi t_{1}\right), \quad u_{m}=\sin \left(\pi t_{1}\right) \sin \left(\pi t_{2}\right) \cdots \sin \left(\pi t_{m-1}\right) \sin \left(\pi t_{m}\right), \\
& u_{r}=\sin \left(\pi t_{1}\right) \sin \left(\pi t_{2}\right) \cdots \sin \left(\pi t_{r}\right) \cos \left(\pi t_{r+1}\right) \quad(1 \leq r \leq m-1) .
\end{aligned}
$$

Then the map $\left(t_{1}, t_{2}, \ldots, t_{m}\right) \mapsto\left[u_{0}: u_{1}: \cdots: u_{m}\right]$ from $I^{m}$ to $\mathbf{P}_{m} \mathbf{R}$ defines, by passage to the quotient, a homeomorphism from $I^{m} / \partial I^{m}$ to $\mathbf{P}_{m} \mathbf{R} / \mathbf{P}_{m-1} \mathbf{R}$ (where $\partial I^{m}$ is the boundary of $I^{m}$ ).

(2) Put $z_{r}=x_{r}+i y_{r}(0 \leq r \leq n)$. Then the map

$$
\left[x_{0}: y_{0}: x_{1}: y_{1}: \cdots: x_{n}: y_{n}\right] \mapsto\left[z_{0}: z_{1}: \cdots: z_{n}\right]
$$

from $\mathbf{P}_{2 n+1} \mathbf{R}$ to $\mathbf{P}_{n} \mathbf{C}$ defines, by restriction and by passage to the quotient, a homeomorphism from $\mathbf{P}_{2 n} \mathbf{R} / \mathbf{P}_{2 n-1} \mathbf{R}$ to $\mathbf{P}_{n} \mathbf{C} / \mathbf{P}_{n-1} \mathbf{C}$.

Combining (1) and (2) and taking $m=2 n$, we thus get a homeomorphism from $I^{2 n} / \partial I^{2 n}$ to $\mathbf{P}_{n} \mathbf{C} / \mathbf{P}_{n-1} \mathbf{C}$, and hence a homeomorphism from $\tilde{\mathscr{C}}\left(\mathbf{P}_{n} \mathbf{C} / \mathbf{P}_{n-1} \mathbf{C} ; Y\right)$ to $\Omega^{2 n}(Y)$.

Acknowledgment. The author thanks Professors S. Oka and M. Kamata, who read a preliminary version of this paper and suggested a number of improvements.

\section{REFERENCES}

[1] D. W. Anderson, A new cohomology theory, Ph. D. Thesis, Univ. of California, Berkeley, 1964.

[2] R. Bott, The stable homotopy of the classical groups, Ann. of Math., (2) 70 (1959), 313-337. 
[3] , Quelques remarques sur les théorèmes de périodicité, Bull. Soc. Math. France, 87 (1959), 293-310.

[4] H. Cartan, Démonstration homologique des théorèmes de périodicité de Bott, Séminaire H. Cartan, 12e année: 1959/60, École Normale Suprérieure, Secrétariat Math., Paris, 1961.

[5] E. Dyer and R. Lashof, A topological proof of the Bott periodicity theorems, Ann. Mat. Pura Appl., (4) 54 (1961), 231-254.

[6] W. Meier, Complex and real K-theory and localization, J. Pure Appl. Algebra, 14 no. 1, (1979), 59-71.

[7] J. Milnor, On spaces having the homotopy type of a CW-complex, Trans. Amer. Math. Soc., 90 (1959), 272-280.

[8] (Morse Theory, Ann. of Math. Studies, No. 51, Princeton Univ. Press, Princeton, N. J., 1963.

[9] R. M. W. Wood, $K$-theory and the complex projective plane, mimeographed notes. ${ }^{4}$

[10] M. Yasuo, On the spaces $\mathrm{O}(4 n) / \mathrm{Sp}$ and $\mathrm{Sp}(n) / \mathrm{O}$, and the Bott maps, Publ. Res. Inst. Math. Sci., 19 no. 1, (1983), 317-326.

[11] (1985), (to appear).

Received March 17, 1986.

YAMANASHI UNIVERSITY

KOFU 400, JAPAN

${ }^{4}$ This is an unpublished paper of Wood, cited in: G. Walker, Quart. J. Math. Oxford (2), 32 (1981), 467-489. 


\title{
PACIFIC JOURNAL OF MATHEMATICS EDITORS
}

\author{
V. S. VARADARAJAN \\ (Managing Editor) \\ University of California \\ Los Angeles, CA 90024 \\ HERBERT Clemens \\ University of Utah \\ Salt Lake City, UT 84112 \\ R. FINN \\ Stanford University \\ Stanford, CA 94305
}

\author{
HERMANN FLASCHKA \\ University of Arizona \\ Tucson, AZ 85721 \\ RAMESH A. GANGOLLI \\ University of Washington \\ Seattle, WA 98195 \\ VAUGHAN F. R. JONES \\ University of California \\ Berkeley, CA 94720 \\ ROBION KIRBY \\ University of California \\ Berkeley, CA 94720
}

\author{
C. C. Moore \\ University of California \\ Berkeley, CA 94720
}

H. SAMELSON

Stanford University

Stanford, CA 94305

HAROLD STARK

University of California, San Diego

La Jolla, CA 92093

\section{ASSOCIATE EDITORS}

\author{
R. ARENS \\ E. F. BECKENBACH \\ B. H. NEUMANN \\ F. WOLF \\ K. YOSHIDA \\ (1906-1982)

\section{SUPPORTING INSTITUTIONS} \\ UNIVERSITY OF ARIZONA \\ UNIVERSITY OF BRITISH COLUMBIA \\ CALIFORNIA INSTITUTE OF TECHNOLOGY \\ UNIVERSITY OF CALIFORNIA \\ MONTANA STATE UNIVERSITY \\ UNIVERSITY OF NEVADA, RENO \\ NEW MEXICO STATE UNIVERSITY \\ OREGON STATE UNIVERSITY

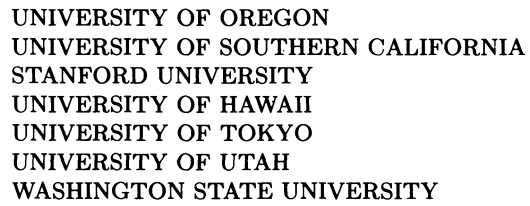

The Supporting Institutions listed above contribute to the cost of publication of this Journal, but they are not owners or publishers and have no responsibility for its content or policies.

Mathematical papers intended for publication in the Pacific Journal of Mathematics should be in typed form or offset-reproduced (not dittoed), double spaced with large margins. Please do not use built up fractions in the text of the manuscript. However, you may use them in the displayed equations. Underline Greek letters in red, German in green, and script in blue. The first paragraph must be capable of being used separately as a synopsis of the entire paper. In particular it should contain no bibliographic references. Please propose a heading for the odd numbered pages of less than 35 characters. Manuscripts, in triplicate, may be sent to any one of the editors. Please classify according to the scheme of Math. Reviews, Index to Vol. 39. Supply name and address of author to whom proofs should be sent. All other communications should be addressed to the managing editor, or Elaine Barth, University of California, Los Angeles, California 90024.

There are page-charges associated with articles appearing in the Pacific Journal of Mathematics. These charges are expected to be paid by the author's University, Government Agency or Company. If the author or authors do not have access to such Institutional support these charges are waived. Single authors will receive 50 free reprints; joint authors will receive a total of $\mathbf{1 0 0}$ free reprints. Additional copies may be obtained at cost in multiples of 50 .

The Pacific Journal of Mathematics is issued monthly as of January 1966. Regular subscription rate: $\$ 190.00$ a year (5 Vols., 10 issues). Special rate: $\$ 95.00$ a year to individual members of supporting institutions.

Subscriptions, orders for numbers issued in the last three calendar years, and changes of address should be sent to Pacific Journal of Mathematics, P.O. Box 969, Carmel Valley, CA 93924, U.S.A. Old back numbers obtainable from Kraus Periodicals Co., Route 100, Millwood, NY 10546.

The Pacific Journal of Mathematics at P.O. Box 969, Carmel Valley, CA 93924 (ISSN 0030-8730) publishes 5 volumes per year. Application to mail at Second-class postage rates is pending at Carmel Valley, California, and additional mailing offices. Postmaster: send address changes to Pacific Journal of Mathematics, P.O. Box 969, Carmel Valley, CA 93924.

PUBLISHED BY PACIFIC JOURNAL OF MATHEMATICS, A NON-PROFIT CORPORATION Copyright (C) 1987 by Pacific Journal of Mathematics 


\section{Pacific Journal of Mathematics}

Vol. 128 , No. $2 \quad$ April, 1987

Pierre Barrucand, John Harold Loxton and Hugh C. Williams, Some

explicit upper bounds on the class number and regulator of a cubic field

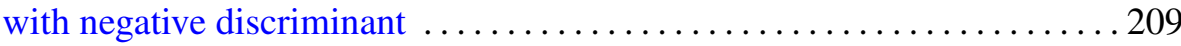

Thomas Ashland Chapman, Piecewise linear fibrations $\ldots \ldots \ldots \ldots \ldots 223$

Yves Félix and Jean-Claude Thomas, Extended Adams-Hilton's

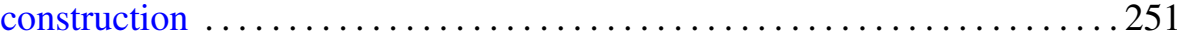

Robert Fitzgerald, Derivation algebras of finitely generated Witt rings . . . 265

K. Gopalsamy, Oscillatory properties of systems of first order linear delay differential inequalities ................................ 299

John P. Holmes, One parameter subsemigroups in locally complete

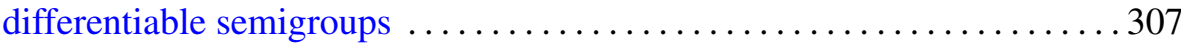

Douglas Murray Pickrell, Decomposition of regular representations for

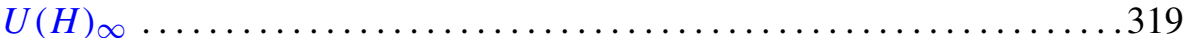

Victoria Powers, Characterizing reduced Witt rings of higher level

Parameswaran Sankaran and Peter Zvengrowski, Stable parallelizability

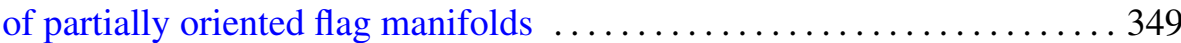

Johan Tysk, Eigenvalue estimates with applications to minimal surfaces . . . 361 Akihito Uchiyama, On McConnell's inequality for functionals of subharmonic functions

Minato Yasuo, Bott maps and the complex projective plane: a construction

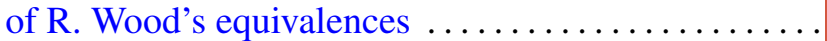

James Juei-Chin Yeh, Uniqueness of strong solutions to stochastic differential equations in the plane with deterministic boundary process 